\title{
Treatment with IMM-101 induces protective CD8+ $T$ cell responses in clinically relevant models of pancreatic cancer
}

\author{
Androulla Elia', Louise Lincoln², Laura Rosa Brunet ${ }^{3}$, Thorsten Hagemann² \\ From Society for Immunotherapy of Cancer 28th Annual Meeting \\ National Harbor, MD, USA. 8-10 November 2013
}

Pancreatic cancer is an aggressive cancer with poor prognosis. Despite its low incidence, it is the 4th cause of cancer-related death in the US. Treatment options have only marginally improved on survival rates, which have remained low, with about $25 \%$ survival at 12 months and $5 \%$ at 5 years. For these reasons, new therapeutic strategies are urgently needed including immunotherapeutic approaches. We have investigated the immunotherapeutic effect of IMM-101, a heat-killed whole cell preparation of Mycobacterium obuense currently undergoing investigation in a Phase II clinical trial in pancreatic cancer (EudraCT n. 2010-022757-42), in two clinically relevant murine models of pancreatic cancer, which histologically mirror human pancreatic adenocarcinomas. Geneticallymodified mice bearing mutations in Kras, p53 and Pdx-Cre (KPC mice) were treated with IMM-101 immediately after development of a palpable tumour. Whereas IMM-101 treatment was unable to effect survival in this rather aggressive model, it did, however, significant decrease metastatic burden. Moreover, it appeared to expand a population of antigen experienced CD8+ T cells bearing CD45RBlowCD44high and able to produce IFN- $\gamma$ and perforin. On the basis of these promising observations, we explored further whether treatment with IMM-101 could induce cytotoxic CD8+ T cells able to effect disease outcome. We treated mice bearing mutations in Kras and Pdx-Cre (KC mice) with IMM-101 and found that not only was survival significantly increased, but also that IMM-101 treatment altered their immune response to disease. We observed systemic $\mathrm{T}$ cell activation at the tumour site, the draining lymph nodes and the spleen, as measured by CD69 expression on T cells. More importantly, in mice treated with IMM-101, CD8+ T cells were found in higher numbers compared to untreated mice, in both the draining lymph nodes and at the tumour site. These CD8+ T cells were characterized by increased production of IFN- $\gamma$, perforin and granzyme, identifying them as cytotoxic CD8+ effector T cells. To further confirm that the mode of action of IMM-101 was directly depended on CD8 $+\mathrm{T}$ cells, we depleted these cells in treated mice with a neutralizing antibody. We found that depletion of CD8+ T cells, but not for example depletion of NK cells, was responsible for the loss of therapeutic effect. We are currently sequencing the CD8+ T cell TCR to determine specificity. We propose that treatment with IMM-101 is able to induce CD8 + T cell-dependent protective effects in the host and limit disease progression. We expect that in combination therapies these immunotherapeutic effects may be further increased.

\section{Authors' details}

${ }^{1}$ St George's, University of London, London, UK. ${ }^{2}$ Barts Cancer Institute, QMUL, London, UK. ${ }^{3}$ Immodulon Therapeutics Ltd, London, UK.

Published: 7 November 2013

doi:10.1186/2051-1426-1-S1-P215

Cite this article as: Elia et al:: Treatment with IMM-101 induces protective CD8+ $T$ cell responses in clinically relevant models of pancreatic cancer. Journal for ImmunoTherapy of Cancer 2013 1(Suppl 1):P215.

${ }^{2}$ Barts Cancer Institute, QMUL, London, UK

Full list of author information is available at the end of the article

(C) 2013 Elia et al; licensee BioMed Central Ltd. This is an Open Access article distributed under the terms of the Creative Commons 